This theorem is a consequence of Theorems $1^{\prime}$ and $4^{\prime}$ and the result of Sierpinski, used by Professor Moore in the proof of Theorem 5.

New York University

\title{
NOTE ON A SCHOLIUM OF BAYES
}

BY F. H. MURRAY

In his fundamental paper on a posteriori probability,* Bayes considered a certain event $M$ having an unknown probability $p$ of its occurring in a single trial. In deriving his a posteriori formula he assumed that all values of $p$ are equally likely, and he recommended this assumption for similar problems in which nothing is known concerning $p$. In the corollary to proposition 8 he derives the value

$$
\int_{0}^{1}\left(\begin{array}{l}
n \\
x
\end{array}\right) p^{x}(1-p)^{n-x} d p=\frac{1}{n+1}
$$

for the probability of $x$ successes in $n$ trials. This result is independent of $x$; in a scholium he observes that this consequence is what is to be expected, on common sense grounds, from complete ignorance concerning $p$, and this concordance is considered to justify the assumption that all values of $p$ are equally likely. $\dagger$

In order to complete the argument of the scholium it is necessary to show that no other frequency distribution for $p$ has the same property.

More precisely, given that a cumulative frequency function $f(p)$ has the property that for $0 \leqq x \leqq n, x, n$ being integers,

$$
\int_{0}^{1}\left(\begin{array}{l}
n \\
x
\end{array}\right) p^{x}(1-p)^{n-x} d f(p)=\frac{1}{n+1}
$$

* Bayes, An essay towards solving a problem in the doctrine of chances, Philosophical Transactions of the Royal Society, vol. 53 (1763), pp. 370-418.

$\dagger$ In other words, the assumption "all values of $p$ are equally likely" is equivalent to the assumption "any number $x$ of successes in $n$ trials is just as likely as any other number $y, x \leqq n, y \leqq n$." It has been suggested verbally by $\mathrm{Mr}$. E. C. Molina that this proposition has a possible importance in certain statistical questions. 
it is required to determine $f(p)$ from this equation. Now if $n=x$, the equation becomes

$$
\int_{0}^{1} p^{x} d f(p)=\frac{1}{x+1}
$$

consequently the moments of $f(p)$ are known. The function $f(p)$ can be completely calculated from these moments with the aid of a theorem of Stieltjes.*

Let

$$
\begin{aligned}
F(z) & =\int_{0}^{1} \frac{d f(p)}{p+z}=\frac{1}{z} \int_{0}^{1} \frac{d f(p)}{1+\frac{p}{z}} \quad(|z|>2) \\
& =\frac{1}{z}\left[\int_{0}^{1} d f-\frac{1}{z} \int_{0}^{1} p d f+\frac{1}{z^{2}} \int_{0}^{1} p^{2} d f-\frac{1}{z^{3}} \int_{0}^{1} p^{3} d f+\cdots\right] .
\end{aligned}
$$

If $f$ is the function already discussed, this becomes

$$
\begin{aligned}
F(z) & =\frac{1}{z}-\frac{1}{2 z^{2}}+\frac{1}{3 z^{3}}-\frac{1}{4 z^{4}}+\cdots \\
& =\log \left(\frac{z+1}{z}\right)
\end{aligned}
$$

Consequently the function $f$ satisfies the equation (for $|z|>2)$

$$
\log \left(\frac{z+1}{z}\right)=\int_{0}^{1} \frac{d f(p)}{p+z} .
$$

From the theorem of Stieltjes, if $\psi(x)$ is a non-decreasing function of $x$, and

then

$$
F(z)=\int_{-\infty}^{\infty} \frac{d \psi(x)}{z+x},
$$

$$
\begin{aligned}
\frac{\psi(\xi-0)+\psi(\xi+0)}{2} & -\frac{\psi(a-0)+\psi(a+0)}{2} \\
& =\lim _{\eta=+0} R\left(\frac{1}{\pi i} \int_{-\xi-i \eta}^{-a-i \eta} F(z) d z\right) .
\end{aligned}
$$

* Stieltjes, Récherches sur les fractions continues, Annales de Toulouse, vol. 8 (1894), pp. 172-175. Also, Perron, Die Lehre von den Kettenbrïchen, p. 372. 
Now the function $F(z)=\log \{(z+1) / z\}$ can be defined on the real axis by continuation, hence the limits above and below the real axis are uniquely determined. Suppose $\xi$, $a$ on the segment $0<a<\xi<1$.

Then

$$
\begin{aligned}
\int_{-\xi-i \eta}^{-a-i \eta}[\log (z+1)-\log z] d z \\
=\int_{-\xi}^{-a}[\log (1+x-i \eta)-\log (x-i \eta)] d x \\
=\left[\begin{array}{c}
(1+x-i \eta) \log (1+x-i \eta)-(1+x-i \eta) \\
-(x-i \eta) \log (x-i \eta)+(x-i \eta)
\end{array}\right]_{-\xi}^{-a} .
\end{aligned}
$$

Now

$$
(1+x-i \eta) \log (1+x-i \eta)-(1+x-i \eta)
$$

approaches real limits, for $x=-a, x=-\xi$, as $\eta \rightarrow 0$, hence makes no contribution to the sum required. We have only to consider

$$
-(-a-i \eta) \log (-a-i \eta)+(-\xi-i \eta) \log (-\xi-i \eta) .
$$

Now as $\eta \rightarrow 0,-\xi-i \eta \rightarrow-\xi$. Since the approach is from below the axis of reals, and since the argument of $\log z$, like that of $\log (1+z)$, is zero for a real positive $z$, the argument here is $-i \pi$. Hence this sum becomes

$$
(a+i \eta)[-\pi i+\log (a+i \eta)]-(\xi+i \eta)[-\pi i+\log (\xi+i \eta)] .
$$

This approaches the limit, as $\eta \rightarrow 0$,

$$
\pi i(\xi-a)+a \log a-\xi \log \xi .
$$

Hence

$$
\lim _{\eta \rightarrow 0} R\left[\frac{1}{\pi i} \int_{-\xi-i \eta}^{-a-i \eta} F(z) d z\right]=\xi-a
$$

Substituting in the identity, we find

$$
\frac{\psi(\xi-0)+\psi(\xi+0)}{2}-\frac{\psi(a-0)+\psi(a+0)}{2}=\xi-a,
$$


or

$$
\frac{\psi(\xi-0)+\psi(\xi+0)}{2}=\xi+\text { const } .
$$

Consequently $\psi$ itself is continuous, $0<\xi<1$.

Now if $a>1, \xi>1$, the integral

$$
\int_{-\xi}^{-a}[\log (z+1)-\log z] d z
$$

is seen to be real, hence

$$
\frac{1}{2}[\psi(\xi-0)+\psi(\xi+0)]-\frac{1}{2}[\psi(a-0)+\psi(a+0)]=0 .
$$

The same is true if both $a$ and $\xi$ are negative.

There are three additive constants yet to be determined, one on each of the intervals $(-\infty, 0),(0,1),(1, \infty)$. If it is assumed that $\psi(-\infty)=0, \psi(+\infty)=1$, and $\psi$ is a non-decreasing function,

$$
\begin{aligned}
\psi(+\infty)-\psi(-\infty)= & 1=\psi(+0)-\psi(-0) \\
& +\psi(1-0)-\psi(+0) \\
& +\psi(1+0)-\psi(1-0)
\end{aligned}
$$

The central term being one, the two remaining terms vanish. Hence $\psi(-0)=\psi(+0)=0, \psi(1+0)=\psi(1-0)=1$. Finally

$$
\psi(\xi)=\left\{\begin{array}{lll}
0, & \text { if } & \xi=0, \\
\xi, & \text { if } & 0<\xi<1, \\
1, & \text { if } & 1<\xi .
\end{array}\right.
$$

American Telegraph and Telephone Company 